\title{
Staging Endometrial Cancer
}

\author{
Jean-Marie Stephan, MD, Megan McDonald, MD, Jean Hansen, DO, Michael J. Goodheart, MD \\ Department of Obstetrics and Gynecology, University of lowa Hospitals and Clinics, lowa City, IA, USA (all authors).
}

\begin{abstract}
Introduction: We report a novel technique for the vaginal placement of a single-incision laparoscopic device to aid in the removal of pelvic and para-aortic lymph nodes in patients undergoing gynecologic cancer surgery.

Technique Description: Informed consent for laparoendoscopic single-site total hysterectomy and bilateral salpingooophorectomy with pelvic and para-aortic lymph node dissection was obtained. A single-incision laparoscopic device was placed through a $2.5-\mathrm{cm}$ umbilical incision, and a total laparoscopic hysterectomy with removal of the ovaries and tubes was performed. Preoperative pathologic analysis showed a grade 2 endometrioid adenocarcinoma of the endometrium, and as a result, bilateral pelvic and para-aortic lymph node dissection was completed. To aid in the lymphadenectomy, an additional transvaginal single-incision laparoscopic device was placed. The procedure was completed in 221 minutes, with 125 minutes spent on the pelvic and para-aortic lymph node dissection. There were no intraoperative or postoperative complications. The amount of blood loss was $50 \mathrm{~mL}$. There were 10 pelvic lymph nodes and 5 para-aortic lymph nodes removed, with no carcinoma detected. The patient tolerated the procedure well and was discharged home the next day.
\end{abstract}

Discussion: Placement of a second transvaginal port is a feasible technique that provides great flexibility and assistance for lymph node removal in gynecologic cancer surgery.

Key Words: Endometrial cancer, Laparoscopic surgery, Lymph node dissection, Laparoendoscopic single-site surgery.

Citation Stephan J-M, McDonald M, Hansen J, Goodheart MJ. Staging endometrial cancer. CRSLS e2014.00246. DOI: 10.4293/CRSLS.2014.00246.

Copyright (c) 2014 SLS This is an open-access article distributed under the terms of the Creative Commons Attribution-Noncommercial-ShareAlike 3.0 Unported

license, which permits unrestricted noncommercial use, distribution, and reproduction in any medium, provided the original author and source are credited.

Address correspondence to: Jean-Marie Stephan, Department of Obstetrics and Gynecology, University of Iowa Hospitals and Clinics, Room 4630 JCP, 200

Hawkins Dr, Iowa City, IA, USA 52242. Telephone: (319) 356-2015, Fax: (319) 353-8363, E-mail: Jean-marie-stephan@uiowa.edu

\section{INTRODUCTION}

In the United States, endometrial cancer is the most common malignancy of the female genital tract. ${ }^{1}$ Most patients present with early-stage disease presumed to be confined to the uterus. Surgical management of early-stage endometrial cancer consists of hysterectomy and bilateral salpingo-oophorectomy, with pelvic and para-aortic lymph node dissection if needed. Recently, with advances in minimally invasive surgery, the concept of single-incision laparoscopic surgery (SILS) has gained interest. Laparoendoscopic single-site surgery (LESS), a term coined by a 2008 consensus statement, uses a single 2- to 3-cm skin incision, usually within the umbilicus, which is in contrast to standard laparoscopic techniques in which multiple small 5- to 10-mm skin incisions are usually required. ${ }^{2}$ There are several challenges encountered when one is attempting lymphadenectomy through a single incision. To overcome the limitations associated with LESS in the context of lymphadenectomy, we present a novel tech- nique that allows for the completion of pelvic and paraaortic lymphadenectomy in select patients who require lymph node removal.

\section{CASE DESCRIPTION}

A 55-year-old woman with postmenopausal bleeding and biopsy-proven grade 2 endometrioid adenocarcinoma of the uterus was taken to the operating room for laparoscopic hysterectomy and bilateral salpingo-oophorectomy, as well as surgical staging. Her medical history included emphysema and controlled hypertension, and her surgical history included a laparoscopic tubal ligation. Her body mass index was $21 \mathrm{~kg} / \mathrm{m}^{2}$.

\section{SURGICAL TECHNIQUE}

The hysterectomy and bilateral salpingo-oophorectomy were performed by a technique similar that described by other surgeons in the literature. ${ }^{3-5}$ After the hysterectomy 
was completed, hemostasis at the vaginal cuff was confirmed, and a similar SILS access device was inserted through the vagina; this allowed the pneumoperitoneum to be maintained (Figure 1). It is important to place the trocars into the SILS access device (Covidien, Mansfield, MA, USA) before its placement into the vagina to avoid any undue injury to the vaginal tissues or bowel. To assist in the pelvic lymph node dissection, 2 articulating graspers were inserted through the vaginal port to help with retraction. One articulating grasper was used to hold open the paravesical or pararectal space, while the other held the peritoneum over the ureter medially (Figure 2). Once adequate exposure was obtained, the surgeon performed the lymph node dissection with 2 devices from the transumbilical port.

Attention was then turned to removing the para-aortic lymph nodes. The peritoneum lateral to the ascending colon was transected to allow for retraction of the colon medially. By use of the vaginal port, a fan was inserted and used to retract the colon and right ureter medially to expose the aorta and inferior vena cava. Another articulating grasper was placed via the vaginal port to assist with retraction of the ureter medially. This allowed exposure of the lymph node packet overlying the inferior vena cava. In a similar manner, after mobilization of the sigmoid colon, a fan was placed through the vaginal port to assist with retraction of the left-sided bowel superiorly and medially. An articulating grasper, placed via the vaginal port, was again used to help retract the ureter medially, thus offering exposure of the left para-aortic lymph node bundle. Adequate exposure with instruments placed through

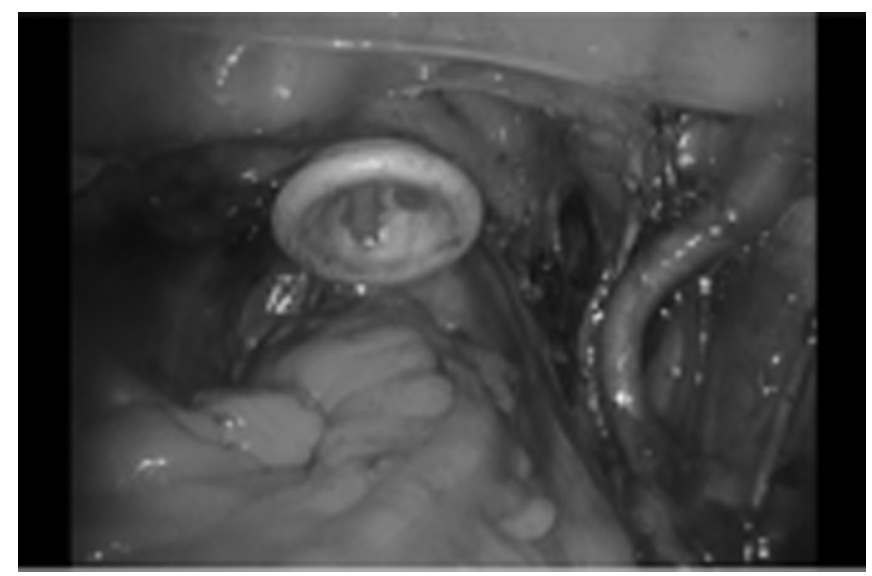

Figure 1. Placement of single-incision laparoscopic device through vaginal cuff. The device maintains the pneumoperitoneum and allows for 3 additional instruments to be placed. (An alternative case is shown to demonstrate port placement.)

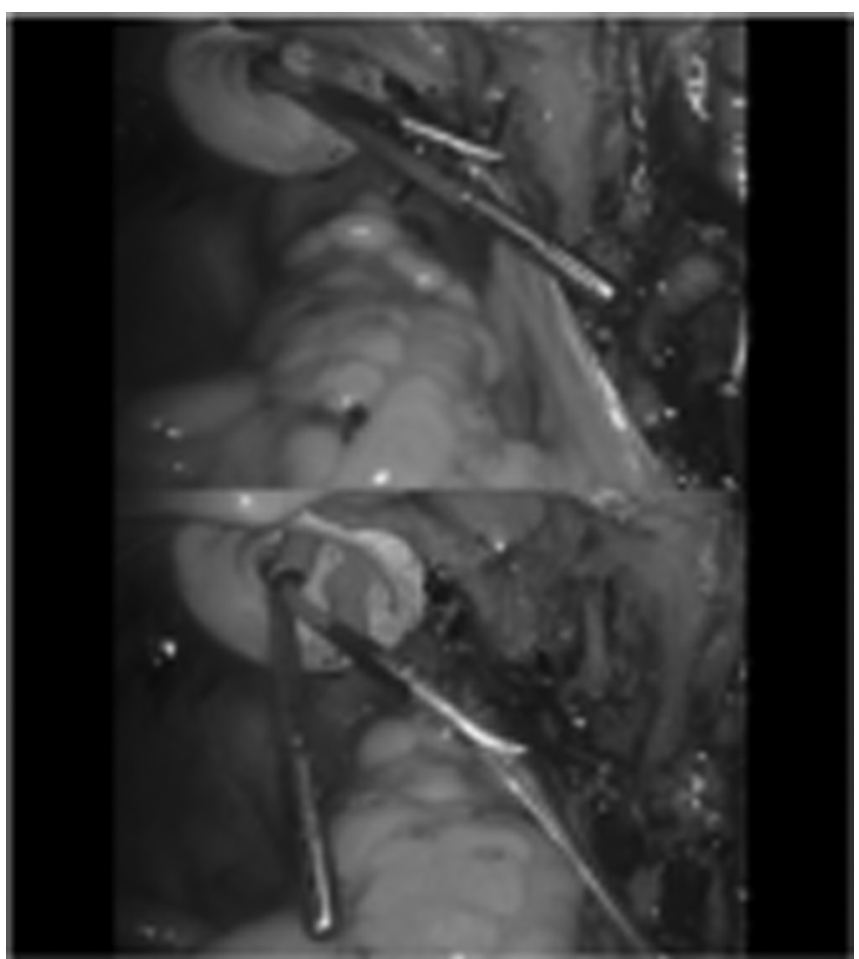

Figure 2. Placement of articulating instruments through vaginal port. (An alternative case is shown to demonstrate instrument placement.)

the vaginal port allowed the operating surgeon to use both umbilical ports to complete the para-aortic lymph node dissection. Pelvic and para-aortic lymph node dissection was performed in accordance with the technique described in the Gynecologic Oncology Group Surgical Procedures Manual. ${ }^{6}$ All lymph node bundles were removed separately through the vaginal port. After the procedure, the vaginal cuff was closed vaginally.

The procedure was completed in 221 minutes, with 125 minutes spent on the pelvic and para-aortic lymph node dissection. There were no intraoperative or postoperative complications. The amount of blood loss was $50 \mathrm{~mL}$, and the patient was discharged home on postoperative day 1 tolerating a regular diet and having return of gastrointestinal function. LESS bilateral lymph node dissection yielded 10 pelvic lymph nodes and 5 para-aortic lymph nodes, with no carcinoma detected in any lymphatic tissue.

\section{DISCUSSION}

The technique of pelvic and para-aortic lymph node dissection by LESS has been reported and was initially described in the literature by Escobar et al. ${ }^{7}$ The technique 
described in this report allows for completion of lymphadenectomy while shortening the learning curve typically associated with LESS.

Having 3 additional trocars placed through the vaginal port to assist with retraction is particularly useful in the obese patient population, in whom performing the paraaortic lymph node dissection can be challenging and timeconsuming. This technique can help provide the additional visualization needed in the setting of adhesions and abundant visceral adipose tissue, as well as in cases in which redundant intra-abdominal tissue prevents adequate exposure. In addition, we believe that this technique offers less chance for hernia formation and less pain because no additional fascial incisions are made, as well as better cosmetic results and improved patient satisfaction scores ${ }^{8}$ when compared with traditional laparoscopy or robotic surgery. Furthermore, after additional cases are performed, we believe that decreased operative times will result because the placement of the vaginal port is quick and easy and allows the placement of up to 3 more laparoscopic instruments. ${ }^{9}$

The novel technique described in this report, which uses the vaginal placement of a single-incision laparoscopic device, can be useful for gynecologic oncologists desiring to learn LESS. This technique allows surgeons to perform endometrial cancer surgery that includes pelvic and paraaortic lymphadenectomy without having to add extra abdominal ports or having to convert to open procedures. Although we plan to incorporate the described technique in our practice and show its feasibility and reproducibility, our vision for the future goes beyond the use of the vaginal port only as an assistant port. In time, we anticipate performing procedures solely via the transvaginal approach. For example, we envision performing a vaginal hysterectomy and bilateral salpingo-oophorectomy followed by a pelvic and para-aortic lymphadenectomy via the vaginal port without a transabdominal camera for patients with endometrial carcinoma. In addition, we would like to be able to offer a completion lymphadenectomy via the transvaginal approach to patients with un- staged endometrial cancer who are referred to us. The spectrum of possibilities is endless, and the door is open for many future exciting cases.

\section{References:}

1. Howlader N, Noone AM, Krapcho M, et al. (eds). SEER Cancer Statistics Review, 1975-2010, National Cancer Institute. Bethesda, MD, http://seer.cancer.gov/csr/1975_2010/, based on November 2012 SEER data submission, posted to the SEER web site, April 2013.

2. Gettman MT, Box G, Averch T, et al. Consensus statement on natural orifice transluminal endoscopic surgery and singleincision laparoscopic surgery: heralding a new era in urology? Eur Urol. 2008;53:1117-1120.

3. Fader AN, Escobar PF. Laparoendoscopic single-site surgery (LESS) in gynecologic oncology: technique and initial report. Gynecol Oncol. 2009;114:157-161.

4. Paek J, Nam EJ, Kim YT, et al. Overcoming technical difficulties with single-port access laparoscopic surgery in gynecology: using conventional laparoscopic instruments. J Laparoendosc Adv Surg Tech A. 2011;21:137-141.

5. Uppal S, Frumovitz M, Escobar P, et al. Laparoendoscopic single-site surgery in gynecology: review of literature and available technology. J Minim Invasive Gynecol. 2011;18:12-23. Philadelphia, PA, Gynecologic Oncology Group.

6. Whitney CW, Spirtos N. Gynecologic Oncology Group Surgical Procedures Manual. 2010;1-41.

7. Escobar PF, Fader AN, Rasool N, et al. Single-port laparoscopic pelvic and para-aortic lymph node sampling or lymphadenectomy: development of a technique and instrumentation. Int J Gynecol Cancer. 2010;20:1268-1273.

8. Song T, Cho J, Kim TJ, et al. Cosmetic outcomes of laparoendoscopic single-site hysterectomy compared with multi-port surgery: randomized controlled trial. J Minim Invasive Gynecol. 2013;20:460-467.

9. Escobar PF, Frumovitz M, Soliman PT, et al. Comparison of single-port laparoscopy, standard laparoscopy, and robotic surgery in patients with endometrial cancer. Ann Surg Oncol. 2012; 19:1583-1588. 